\title{
Evaluación de las actitudes e intereses hacia la lectura: validación de un instrumento para lectores principiantes
}

\author{
Teresa Artola ${ }^{1}$, Santiago Sastre ${ }^{1}$ y Jesús María Alvarado ${ }^{2}$ \\ ${ }^{1}$ C.U. Villanueva, Universidad Complutense de Madrid (España); \\ ${ }^{2}$ Universidad Complutense de Madrid (España)
}

\begin{abstract}
Se presenta un instrumento de evaluación de las actitudes e intereses lectores destinado a estudiantes de los primeros cursos de Primaria, que se deriva de la escala elaborada por Meirisuo-Storm y Soininen (2012). La actitud y la motivación pueden ser dos de las principales razones por las que la mayoría de los lectores leen: no obstante, a menudo se ha ignorado el importante papel que éstas desempeñan. El cuestionario evalúa cinco dimensiones: motivación hacia la lectura, intereses lectores, actitudes hacia la lectura social, percepción de la competencia lectora y motivación hacia el aprendizaje y el estudio. Se ha llevado a cabo un análisis factorial confirmatorio con una muestra de 1135 estudiantes de $2^{\circ}$ de Primaria, con una edad de entre 7 y 8.4 años (media 7.7 y $D T=0.99$ ). Dicho análisis muestra un buen ajuste del modelo de cinco factores a los datos. Asimismo, se han realizado los estudios de invarianza exigibles para que el instrumento pueda aplicarse en niños y niñas de $2^{\circ}$ de Primaria. Tras eliminar las fuentes de invarianza, el análisis muestra algunas diferencias de género significativas: las niñas presentan una mayor motivación hacia la lectura y una actitud más positiva hacia la lectura social, mientras que los niños muestran una mejor percepción de la competencia lectora. Además, se observan importantes diferencias en los intereses lectores. Se concluye que este instrumento puede servir de gran ayuda al profesorado para estimar los niveles de actitud y motivación del alumnado de forma fiable, así como para la evaluación de estudiantes específicos y para la estimación de los cambios que se producen en las actitudes tras programas de intervención.
\end{abstract}

Palabras Clave: Actitudes lectoras, intereses lectores, motivación a la lectura, lectura social, diferencias de género y Educación Primaria.

Reading Attitudes and Interests Questionnaire. This article presents an instrument for the evaluation of reading attitudes and interests of Spanish students who have just started to read, that derives from the scale developed by Meirisuo-Storm \& Soinien (2012). Attitude and motivation can be one of the main reasons why most readers read and possibly the main reason why many do not read. The important role that they play, however, has often been ignored. The questionnaire assesses five dimensions related to reading: motivation towards reading, reader's interests, attitudes toward social reading, perception of reading competence and motivation towards learning or study. A confirmatory factor analysis was carried out with a sample of 1135 second graders, with an age between 7 and 8.4 years old (median 7.7 and standard derivation=0.99). This analysis shows a good fit of the five factor model to the data. Also invariance studies have been done to prove that the instrument can be applied without significant biases to $2^{\text {nd }}$ grade boys and girls. Once the main sources of invariance were eliminated, the analysis showed some significant sex differences: girls showed greater motivation towards reading and more positive attitudes toward social reading, while boys showed a better perception of their reading competence. Also interesting differences in reader's interests were observed. It is concluded that this instrument can be useful as it can help teachers estimate levels of attitude and motivation of their students in a reliable manner, as well as for the evaluation of specific students and for the estimation of the changes that occur in attitudes following intervention programmes.

Key words: Reading attitude, reading enjoyment, reading interests, reading aloud, gender differences and Primary Education.

Correspondencia: Santiago Sastre Llorente. Centro Universitario Villanueva. Universidad Complutense de Madrid. Departamento de Psicopedagogía. C/ Costa Brava, nº 2. C.P.: 28034. Madrid (España). E-mail: ssastre@villanueva.edu 
ARTOLA, SASTRE y ALVARADO. Cuestionario de Actitudes e Intereses Lectores 
La actitud hacia la lectura puede definirse como el conjunto de sentimientos que pueden conducir a las personas a aproximarse o rechazar esta actividad (Cooter y Alexander, 1984). Los sentimientos positivos o negativos que los niños y las niñas tienen hacia la lectura están estrechamente relacionados con su éxito en ella y, dado que estas actitudes se desarrollan en la primera infancia, los primeros años escolares parecen ser cruciales (Cartwright, Marshall y Wray, 2015; Stutz, Schaffner y Schiefele, 2016), puesto que las habilidades lectoras van a tener un importante peso en el rendimiento académico de los estudiantes (Elosua y Gómez-Veiga, 2014). Por todo ello, “es importante diseñar e implementar programas que fomenten la lectura temprana, formando hábitos y una motivación para leer en los jóvenes lectores" (Aguilar, Aragón, Navarro, Delgado y Marchena, 2017, p. 31).

Los resultados de numerosos estudios indican que, en muchos países de la Unión Europea, las niñas obtienen mejores resultados en su lectura que los niños (Merisuo-Storm y Soininen, 2012; Stevens, 2011; Villalón, Meirsuo-Storm e Izquierdo-Magaldi, 2015; Watson, Kehler y Martino, 2010), especialmente en la comprensión de textos narrativos (Schwartz, 2002), y que estas diferencias pueden afectar negativamente al rendimiento académico de los chicos (Sadowski, 2010). Para interpretar estas diferencias se han utilizado diversas hipótesis, entre las que destacan las posibles discrepancias en las actitudes, la motivación y, fundamentalmente, los intereses, valores, objetivos y actividades extraacadémicas.

La importancia de la actitud y de la motivación con la que se aborda la lectura es evidente y la actitud de un estudiante hacia la lectura es uno de los factores que más influencia tiene sobre su rendimiento lector. No obstante, como indican McKenna y Kear (1990), la mayor parte de los estudios se han centrado más en evaluar la comprensión lectora y son escasas las investigaciones que han abordado la relación entre la competencia lectora y las percepciones y actitudes de los lectores principiantes, en especial cuando se trata de lectores de países de habla hispana (Villalón, Melero e Izquierdo-Magaldi, 2014). Merisuo-Storm (2006) constata en sus investigaciones diferencias en las actitudes hacia la lectura de niños y niñas de entre diez y once años. Estas diferencias se observan igualmente en edades tempranas, cuando todavía están iniciándose en la lectura (Graham, Berninger y Abbott, 2012; Marinak y Gambrell, 2010; Merisuo-Storm y Soininen, 2012). Estos estudios indican que las niñas disfrutan más de la lectura que los niños; otros (Sadowski, 2010; Sullivan, 2004) señalan que los chicos, generalmente, leen menos que las chicas, lo que suele afectar a su fluidez lectora, y que muchos de estos tienden a percibirse como peores lectores que las chicas. Asimismo, algunos autores hablan de diferencias entre sexos en los intereses lectores (Artola, Sastre y Barraca, 2017; Boltz, 2007).

Villalón, Melero e Izquierdo-Magaldi $(2014,2016)$ han llevado a cabo una investigación similar sobre las actitudes hacia la lectura con estudiantes españoles de los 
dos primeros cursos de Primaria, en los que los estudiantes están todavía aprendiendo a leer y escribir. Para ello utilizaron una versión adaptada al castellano del cuestionario de actitudes e intereses lectores elaborado en Finlandia por Merisuo-Storm (2006). En este estudio se analiza la relación que las actitudes e intereses lectores guardan con la comprensión lectora y con el sentimiento de competencia como lector de los sujetos. Los resultados revelan que los niños y niñas españoles muestran una buena actitud e interés hacia la lectura y se consideran bastante competentes en ella, coincidiendo así con lo encontrado por Merisuo-Storm y Soininen (2012) con niños finlandeses. No obstante, se observan algunas diferencias interesantes entre sexos: las niñas presentan una actitud más positiva hacia la lectura. Estas diferencias de género ya han sido encontradas en estudios previos en diversas edades y en distintos contextos geográficos (Artola et al., 2017; Graham, Berninger y Abbott, 2012; Merisuo-Storm, 2006; Merisuo-Storm y Soininen, 2014; Mullis, Martin, Foy y Drucker, 2011) y parecen observarse ya desde edades tempranas (Villalón et al., 2014). Por otra parte, varios estudios apuntan hacia el hecho de que se produce una disminución del interés y la motivación hacia la lectura a medida que los niños crecen y que los varones empiezan a perder el interés hacia la lectura en edades tempranas (Merisuo-Storm, 2006; Pestcher, 2010).

También algunas investigaciones informan de diferencias de género en los intereses lectores (Chick y Heilman-Houser, 2000; Oakhill y Petrides, 2007). Los niños suelen elegir un tipo de libros diferente que las niñas (Boltz, 2007; Mohr, 2006): las niñas suelen preferir narraciones en las que aparecen temas relacionados con la familia y la amistad; mientras que los chicos habitualmente quieren leer textos más realistas y no de ficción (deporte, ciencias, historia), así como lecturas de textos informativos breves (cómics, manuales de cómo hacer cosas, libros con mucha acción o de terror) (Artola et al., 2017; Merisuo-Storm, 2006; Schwartz, 2002; Sullivan, 2004). Muchos chicos no muestran interés hacia los libros que suelen encontrarse en las bibliotecas del aula: habitualmente, el tipo de lecturas que les gusta no se encuentra en ellas.

En definitiva, los estudios revisados sugieren que existen diferencias en los intereses lectores entre niños y niñas, y que el rendimiento cognitivo de los varones se ve más afectado que el de las chicas por la actitud y el interés hacia el tema abordado. Las niñas es más probable que persistan en la lectura que los chicos, incluso aunque el texto no sea de su interés (Ainley, Hillman y Hidi, 2002). Además, los chicos se ven más influidos, a la hora de elegir los libros, por las expectativas y los estereotipos de género que las niñas, en especial cuando se encuentran en público (Chapman, Filipenko, McTavish, y Saphiro, 2007; Mohr, 2006).

Asimismo, parece que a la mayoría del alumnado no le agrada la lectura en voz alta, pero esto es así especialmente en los chicos que, incluso aunque sean lectores competentes, tienden a sentirse avergonzados cuando leen en público 
(Merisuo-Storm, 2006). Finalmente, investigaciones realizadas sobre la motivación y la actitud hacia la lectura en etapas tempranas $\left(1^{\circ}\right.$ y $2^{\circ}$ de Primaria) indican que existe una estrecha relación entre el rendimiento lector y la percepción de la competencia lectora (Cartwright, Marshall y Wray, 2015).

Todos estos estudios indican que, dada la importancia que la lectura tiene para el rendimiento en muchas áreas del currículum, parece importante buscar formas de mejorar la actitud y los intereses lectores. Esto es especialmente relevante en los varones, desde edades tempranas.

En esta investigación se presentan los resultados de un estudio sobre actitudes e intereses lectores realizado con estudiantes españoles de $2^{\circ}$ de Primaria. Previo a la exploración de los resultados, se ha llevado a cabo un análisis para descartar la posible existencia de sesgos o falta de equidad del cuestionario al comparar los grupos. El cuestionario administrado es una adaptación del utilizado con alumnado finlandés por Merisuo Storm y Soininen (2012), y con estudiantes españoles por Villalón et al. (2014), si bien en estos estudios previos no se analizó la estructura factorial del cuestionario ni la adecuación de la medida a los distintos grupos sometidos a comparación. En esta investigación se incluye, además, una nueva variable relacionada con la actitud hacia el aprendizaje y el estudio.

El objetivo primordial de esta investigación es realizar una validación del instrumento, identificando en primer lugar la estructura factorial del mismo y, una vez establecida, realizar los estudios de invarianza exigibles para que el instrumento pueda aplicarse a estudiantes de $2^{\circ}$ de Primaria. En concreto, se pondrán a prueba los modelos de invarianza de configuración, métrica, escalar y estricta: una vez que se concluye que la estructura factorial del constructo es igual en ambos grupos (invarianza de configuración), el primer nivel de invarianza es la métrica en la que se exige que los pesos factoriales sean iguales en ambos grupos (invarianza débil), esto es, que la medida de los distintos ítems sea igual de discriminante para ambos grupos. Un nivel de invarianza superior es el de invarianza escalar, en la que además de los niveles de invarianza anteriores, se exigen iguales interceptos para los distintos ítems. Esta varianza se denomina como fuerte, ya que una vez alcanzada son legítimas las comparaciones entre sujetos de los distintos grupos, ya que se asegura que las puntuaciones de los sujetos, independientemente del grupo, serán iguales para niveles equivalentes en la variable latente. El mayor nivel de invarianza se logra cuando los residuales o términos error también son iguales.

Los estudios sobre las posibles fuentes de invarianza y los posibles sesgos de los tests, o de algunos de sus ítems, son necesarios si queremos garantizar la equidad y la validez de las interpretaciones que de estos estudios se derivan. Sin estos estudios, no podemos tener la certeza de que las diferencias encontradas se deban realmente a la existencia de diferencias reales entre los grupos o al sesgo de determinados ítems. 
Por ello, en este estudio también nos preguntamos si las diferencias entre sexos en las actitudes e intereses lectores, encontradas en estudios previos, son debidas a la existencia real de estas diferencias entre los sexos o son debidas a artefactos generados por el propio instrumento de medida empleado, al existir sesgos en algunos de los ítems que componen el test.

Por tanto, pretendiendo salvar esos posibles problemas, perseguimos en este estudio los siguientes objetivos:

- Validar un instrumento de medida de las actitudes e intereses lectores en estudiantes españoles de los primeros cursos de Primaria.

- Investigar las actitudes y motivación hacia la lectura, así como los intereses lectores, de niños y niñas españoles al comenzar la Educación Primaria, y analizar si algunos de estos aspectos difieren realmente en función del género o son simplemente el resultado artificial de los sesgos en el instrumento de medida.

- Describir cómo los niños y niñas de segundo de Primaria perciben su nivel de competencia lectora y si existen diferencias de género en la apreciación de dicha competencia.

- Revelar las posibles diferencias entre niños y niñas de $2^{\circ}$ de Primaria en cuanto a la actitud hacia el aprendizaje y el estudio.

\section{MÉTODO}

\section{Participantes}

Tras traducir y adaptar el cuestionario y someterlo a la validación por medio del juicio de expertos, se procedió a su aplicación colectiva a una muestra de 1135 sujetos, con una edad de entre 7 y 8.4 años (media 7.7 y $D T=0.99), 565$ niños $(49.77 \%$ ) y 570 niñas $(50.22 \%)$. Todos ellos cursan $2^{\circ}$ de Primaria en quince centros concertados y privados de la comunidad de Madrid.

\section{Procedimiento}

El muestreo utilizado es no probabilístico por conveniencia: los investigadores seleccionaron directamente quince centros educativos con los que se mantenía alguna relación y que accedieron a la colaboración. Tras obtener la autorización de dichos colegios, y tras informarles detalladamente de los objetivos que se pretendían con este estudio, se procedió a aplicar colectivamente la prueba, siempre en horario escolar y en presencia de los investigadores.

Se solicitó y obtuvo el permiso a los autores del cuestionario original para adaptarlo y publicarlo. Además, todo este proceso se realizó respetando las normas éticas recogidas en el código deontológico de Psicología. 


\section{Instrumentos}

El instrumento que se pretende validar en este estudio fue elegido por ajustarse a los objetivos que se pretendían en la investigación. Se trata de una adaptación al castellano del cuestionario utilizado en un estudio llevado a cabo en Finlandia (Merisuo-Storm y Soininen, 2012). El cuestionario evalúa, por medio de 22 ítems y con una escala tipo Likert de 1 a 4 (donde 1 se corresponde a "me encanta" o "me resulta muy fácil", y 4 a "lo odio" o "me resulta muy difícil") cuatro dimensiones: motivación hacia la lectura, intereses lectores, actitudes hacia la lectura social y percepción de la competencia lectora. Además, se incluyeron 4 ítems más destinados a profundizar en una nueva dimensión de especial interés, la actitud hacia el aprendizaje y el estudio (Tabla 1).

Tabla 1. Ítems comprendidos en cada dimensión evaluada
\begin{tabular}{lc}
\multicolumn{1}{c}{ Dimensiones } & \multicolumn{1}{c}{ Ítems } \\
\hline Motivación hacia la lectura & $1,3,4,11,12$ \\
\hline Intereses lectores & $2,5,6,7,8,9,10$ \\
\hline Actitudes hacia el estudio & $13,14,15,16$ \\
\hline $\begin{array}{l}\text { Actitudes hacia la lectura } \\
\text { social o colectiva }\end{array}$ & $17,18,19,20,21$ \\
\hline $\begin{array}{l}\text { Percepción de la competencia } \\
\text { lectora }\end{array}$ & $22,23,24,25,26$ \\
\hline
\end{tabular}

\section{Análisis de datos}

En primer lugar se analizó la consistencia interna del instrumento, mostrando un coeficiente $\alpha$ de .89; sin embargo, al ser un instrumento multidimensional un estimador más adecuado es omega jerárquico, que fue de .63 (ver Green y Yang, 2015; Trizano-Hermosila y Alvarado, 2016) indicando que el 63\% de la varianza del test se debe a un factor general, con las siguientes fiabilidades para cada escala: motivación hacia la lectura, .70; intereses lectores, .57; actitud hacia el estudio, .74; actitud hacia la lectura social o colectiva, .74; y percepción de la competencia lectora, .78.

Para la validación del instrumento y para realizar los estudios de invarianza, se han utilizado las técnicas de análisis factorial recomendadas para datos categóricos y ordinales (Asún, Rdz-Navarro y Alvarado, 2016) realizados a partir de la matriz de correlaciones policórica y utilizando como método de estimación Mínimos Cuadrados Ponderados Diagonalmente (Diagonally Weighted Least Squares, DWLS). En los análisis factoriales confirmatorios se aplicó la corrección Satorra-Bentler para la estimación de $\chi^{2}$, y para el cálculo de las diferencias en $\chi^{2}$ en los análisis factoriales confirmatorios multigrupo, la corrección de Satorra (2000). Se utilizaron los paquetes estadísticos SPSS (v. 22), Lisrel (v. 8.80) y R (Psych, Lavaan y semTols). 


\section{RESULTADOS}

\section{Estructura Factorial del cuestionario}

Para conocer la dimensionalidad del test se aplicó la técnica de análisis paralelo. El análisis paralelo indica la existencia de cinco componentes, que en conjunto explicarían el $53.91 \%$ de la varianza (primer autovalor $=6.88$ explica=27.52\%, segundo autovalor $2.54=10.16 \%$, tercer autovalor $1.62=6.50 \%$, cuarto autovalor $1.26=5.06 \%$ y quinto autovalor $1.17=4.67 \%$ ).

A partir del resultado del análisis paralelo se decide realizar un Análisis Factorial Confirmatorio (AFC) para conocer la bondad de ajuste al modelo de cinco factores propuesto. El AFC reveló que la bondad de ajuste al modelo de cinco factores es aceptable como muestran los siguientes índices: $\chi^{2}(289)=1371.94 ; p<0.001$, el índice de ajuste comparativo $\mathrm{CFI}=0.95$, el índice de ajuste no normalizado $\mathrm{NNFI}=0.95$, la media cuadrática del error de aproximación RMSEA $=0.06$, la media cuadrática del residual estandarizado SRMR=0.08. Salvo el valor de $\chi^{2}$ cuadrado que tiende a desajustar en tamaños muestrales grandes, los demás índices se encuentran dentro de los puntos de corte recomendados por $\mathrm{Hu}$ y Bentler (1999), que deben ser >.95/.90 para CFI y NNFI $<.05 / .08$ para RMSEA, y <.06/.08 para SRMR para un ajuste bueno o aceptable, respectivamente.

No obstante, al revisar los pesos factoriales se observó que el ítem 10 (¿Te gusta que los personajes de los cuentos sean malvados y den miedo?) tenía un peso muy bajo $0.10(t=1.96 ; p=0.05)$ por lo que se decidió eliminarlo del análisis, observándose una mejora en la bondad de ajuste del modelo: $\chi^{2}(265)=1078.50 ; p<0.001$; $\mathrm{CFI}=0.96$; NNFI=0.96; RMSEA=0.05 y SRMR=0.07.

A continuación se evaluó la bondad de ajuste para cada grupo, observándose una bondad de ajuste similar al modelo cinco factores.

En niños $\chi^{2}(265)=635.60 ; p<0.001 ; \mathrm{CFI}=0.97 ; \mathrm{NNFI}=0.96 ; \mathrm{RMSEA}=0.050 \mathrm{y}$ $\mathrm{SRMR}=0.074 . \quad$ En niñas $\chi^{2} \quad(265)=669.42 ; \quad p<0.001 ; \quad \mathrm{CFI}=0.96 ; \quad \mathrm{NNFI}=0.96$; RMSEA=0.05 y SRMR=0.08 (Figura 1). 

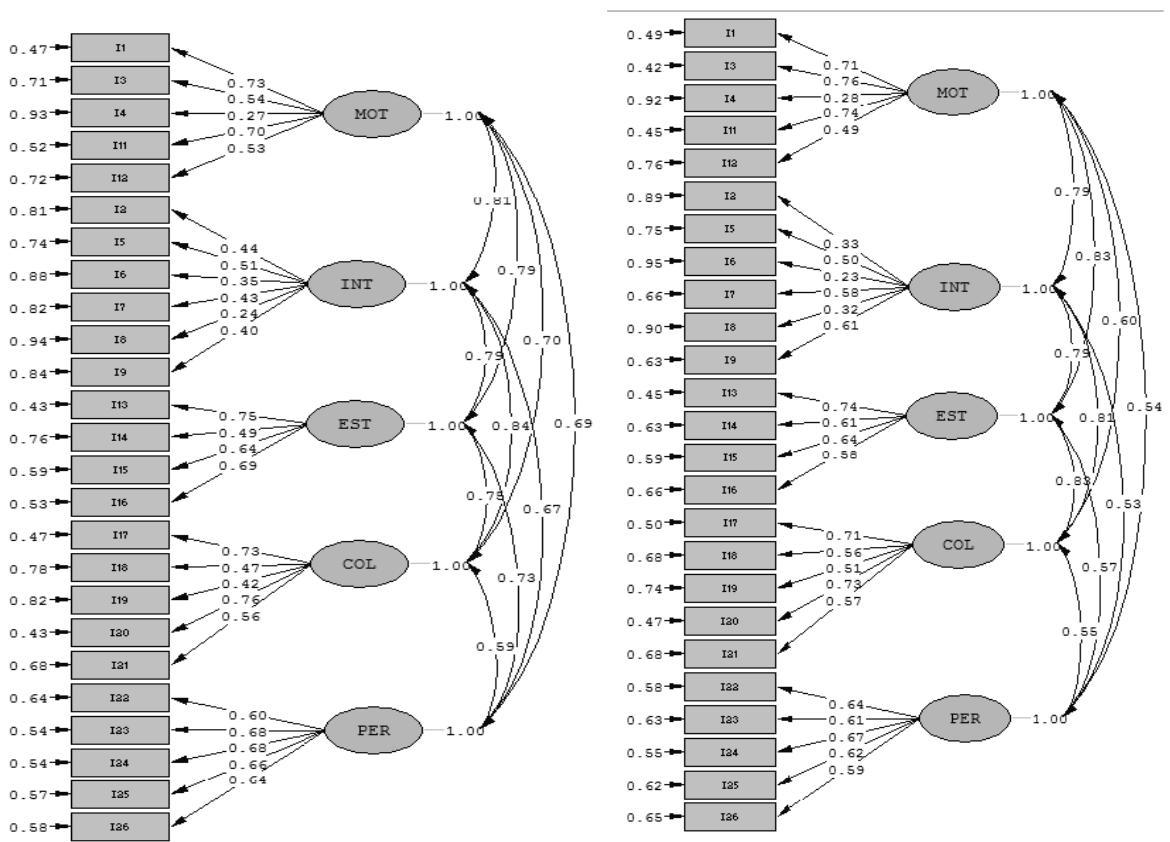

Figura 1. Path diagram del CFA para el grupo de niñas (izquierda) y de niños (derecha)

\section{Estudios de Invarianza}

La tabla 2 nos revela que el instrumento original (eliminado el ítem 10) no sería aplicable en muestras de niños y niñas españoles, ya que no alcanza el nivel mínimo de invarianza: la diferencia en chi-cuadrado es estadísticamente significativa $(p<0.05)$ y además se observa un incremento de una décima en CFI respecto al modelo de configuración.

Tabla 2. Invarianza en relación al sexo

\begin{tabular}{lccccccc}
\hline & $\chi^{2}$ & $g l$ & $\chi^{2}$ dif. & $g l$ & $p$ & CFI & RMSEA \\
\hline Configuración & 1265.77 & 530 & & & & 0.96 & 0.05 \\
\hline Débil & 1405.30 & 550 & 34.54 & 15.04 & $<0.01$ & 0.95 & 0.05 \\
\hline Fuerte & 1784.59 & 595 & 144.21 & 28.47 & $<0.01$ & 0.93 & 0.06 \\
\hline Estricta & 1838.07 & 600 & 16.69 & 2.97 & $<0.01$ & 0.92 & 0.06 \\
\hline
\end{tabular}

Mediante el estudio de los índices de modificación se observa que los ítems con mayor desajuste son el 2 (¿Te gusta leer tebeos o cómics?) y 3 (¿Te gusta que te regalen libros?). Es por esto por lo que se procede a evaluar la invarianza sin ellos (Tabla 3). 
Tabla 3. Invarianza en relación al sexo eliminados ítems 2 y 3

\begin{tabular}{lccccccc}
\hline & $\chi^{2}$ & $g l$ & $\chi 2$ dif. & $g l$ & $p$ & CFI & RMSEA \\
\hline Configuración & 1086.27 & 440 & & & & 0.95 & 0.05 \\
\hline Débil & 1161.21 & 458 & 21.49 & 14.72 & 0.11 & 0.95 & 0.05 \\
\hline Fuerte & 1326.35 & 499 & 64.32 & 26.83 & $<0.01$ & 0.94 & 0.06 \\
\hline Estricta & 1423.67 & 504 & 32.80 & 3.05 & $<0.01$ & 0.94 & 0.06 \\
\hline
\end{tabular}

Nota. Método de estimación DWLS aplicando la corrección de Satorra (2000) para el cálculo de las diferencias en chi-cuadrado.

Una vez alcanzado el nivel de invarianza débil, se identificó a partir de los índices de modificación que los ítems que impiden obtener invarianza fuerte son los ítems 4 (¿Te gusta que te lean libros en voz alta?) y 6 ( ¿Te gusta leer libros que no sean de cuentos?) (Tabla 4).

Tabla 4. Invarianza en relación al sexo eliminados ítems 2, 3, 4 y 6

\begin{tabular}{lccccccc}
\hline & $\chi^{2}$ & $g l$ & $\chi^{2}$ dif. & $g l$ & $p$ & CFI & RMSEA \\
\hline Configuración & 723.66 & 358 & & & & 0.97 & 0.04 \\
\hline Débil & 771.67 & 374 & 15.37 & 13.45 & 0.32 & 0.97 & 0.04 \\
\hline Fuerte & 829.27 & 411 & 22.64 & 24.72 & 0.58 & 0.97 & 0.04 \\
\hline Estricta & 935.16 & 416 & 36.76 & 3.10 & $<0.01$ & 0.96 & 0.05 \\
\hline
\end{tabular}

Nota. Método de estimación DWLS aplicando la corrección de Satorra (2000) para el cálculo de las diferencias en chi-cuadrado.

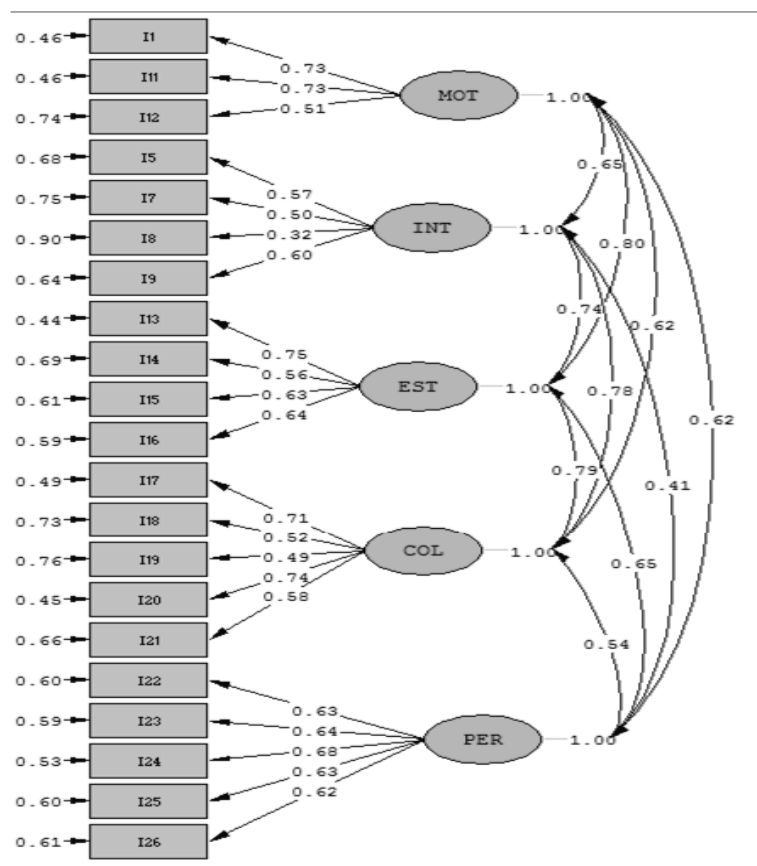

Figura 2. Path diagram modelo final 
Una vez eliminadas las fuentes de invarianza, la bondad de ajuste del modelo final ha mejorado. Así lo muestran los índices chi-cuadrado (179)=617.20; $p<0.001$; $\mathrm{CFI}=0.98$; NNFI=0.97; RMSEA=0.05 y $\mathrm{SRMR}=0.06$ (Figura 2).

\section{Diferencias entre los grupos}

La invarianza escalar nos garantiza que podemos comparar entre integrantes de ambos grupos, ya que si dos sujetos tienen el mismo nivel en la variable subyacente les corresponderá igual puntuación observada. En consecuencia, podemos hacer una comparación legítima o justa y ver si en las distintas medidas hay diferencias entre los grupos (están formados por individuos con distinto nivel en la variable latente o constructo medido) (Tabla 5).

Tabla 5. Medias y desviaciones típicas en cada grupo para las distintas medidas, y pruebas de significación de igualdad de varianzas y de medias mediante la $t$ de Student y para el tamaño del efecto la $d$ de Cohen

(valores en torno a 0.5 indican un efecto medio)

\begin{tabular}{ccccccccccc}
\hline & \multicolumn{1}{c}{ NIÑOS } & \multicolumn{1}{c}{ NIÑAS } & \multicolumn{7}{c}{ Pruebas de significanción } \\
\hline MEDIDA & $M$ & $D T$ & $M$ & $D T$ & $F$ & $p$ & $t$ & $g l$ & $p$ & $d$ \\
\hline MOTIVACIÓN & 5.09 & 1.96 & 4.85 & 1.88 & 1.76 & 0.185 & 2.12 & 1100 & 0.034 & 0.17 \\
\hline INTERESES & 6.64 & 2.30 & 5.75 & 1.82 & 34.93 & $<0.001$ & 7.09 & 1045 & $<0.001$ & 0.62 \\
\hline ESTUDIOS & 6.28 & 2.27 & 6.33 & 2.23 & 0.01 & 0.988 & -0.38 & 1100 & 0.702 & 0.03 \\
\hline SOCIAL & 8.84 & 3.23 & 8.04 & 2.69 & 23.18 & $<0.001$ & 4.50 & 1065 & $<0.001$ & 0.46 \\
\hline PERCEPCIÓN & 7.97 & 2.62 & 8.53 & 2.8 & 2.68 & 0.102 & -3.41 & 1100 & 0.001 & 0.34 \\
\hline TOTAL & 34.82 & 8.82 & 33.50 & 8.20 & 3.60 & 0.06 & 2.59 & 1100 & 0.010 & 0.45 \\
\hline
\end{tabular}

Nota. Las escalas evalúan las variables en dirección inversa (a mayor puntuación, menor motivación, interés, percepción).

La tabla 5 revela que hay una diferencia estadísticamente significativa $(p=0.01)$ entre los niños y las niñas en la puntuación global, esto es, las niñas obtienen una mejor puntuación (menor puntuación indica un mayor nivel) que los niños. Este resultado se observa tanto en "motivación hacia la lectura" como en "intereses lectores" y "actitud hacia la lectura colectiva o social". En la variable "actitud hacia el aprendizaje y el estudio" no se observan diferencias entre ambos grupos, y la relación se invierte en "percepción de la competencia lectora". Es decir, la "actitud hacia el aprendizaje y el estudio" es similar en niños y niñas, pero mientras que ellas muestran una mayor motivación e interés y les gusta más la lectura en grupo, los niños valoran su nivel de competencia lectora por encima de lo que lo hacen las niñas.

\section{DISCUSIÓN}

La lectura constituye una competencia fundamental para el rendimiento escolar y para la vida diaria, cuyo logro depende en gran medida de una actitud positiva hacia la misma. Como ya indicó el filósofo Rousseau, cualquier método para enseñar puede ser adecuado si contamos con una actitud positiva por parte del que aprende, y 
como defienden Anderson, Hiebert, Scott y Wilkinson (1985, citados por McKenna y Kear, 1990) convertirse en un lector eficaz pasa por descubrir que el material escrito puede ser interesante. No obstante, los estudios sobre cómo mejorar el rendimiento lector a menudo han ignorado el importante papel que desempeñan las actitudes e intereses lectores en el proceso de convertirse en un lector eficiente.

Con este estudio hemos perseguido adaptar y validar un instrumento que puede permitir al profesorado estimar de forma fiable las actitudes e intereses lectores de estudiantes de los primeros cursos de Primaria. Se presenta la adaptación de este instrumento para población española, así como el análisis y eliminación de las posibles fuentes de invarianza del mismo.

Como indican Gómez-Benito, Hidalgo y Guilera (2010), la existencia de sesgos en los instrumentos de medida representa una importante amenaza contra la validez de las conclusiones que de ellos se derivan. Contrariamente, demostrar un alto nivel de invarianza en el test permite sostener que las diferencias entre los sexos encontradas en la investigación se dan realmente. Estos análisis son especialmente importantes cuando se adaptan tests existentes a otras lenguas y culturas o a otras poblaciones diferentes a aquellas utilizadas cuando se construyó la prueba.

Los resultados nos permiten afirmar que este instrumento puede aplicarse a estudiantes de Primaria, por tener un alto nivel de consistencia interna, así como una adecuada estructura factorial, una vez eliminado el ítem 10, que se ajusta al modelo de cinco factores propuesto.

En cuanto a los estudios de invarianza, se han puesto a prueba los modelos de invarianza de configuración, métrica, escalar y estricta. El análisis de los datos indica que algunos ítems del cuestionario original muestran niveles de dificultad y/o discriminación diferentes para niños y niñas, pero la eliminación de cuatro de ellos ( 2 y 6 , relacionados con los intereses lectores, y 3 y 4, relacionados con la motivación hacia la lectura recreativa o informal), permite obtener un nivel de invarianza fuerte, lo que admite hacer comparaciones legítimas, ya que se asegura que las puntuaciones de los sujetos, independientemente del grupo, serán iguales para niveles equivalentes en la variable latente. Así, el cuestionario definitivo queda configurado por 21 ítems que evalúan cinco dimensiones relacionadas con la lectura en los primeros cursos de Primaria: motivación hacia la lectura, intereses lectores, actitudes hacia la lectura social, percepción de la competencia lectora y actitud hacia el aprendizaje y el estudio.

Una vez garantizada la invarianza escalar, se ha procedido a las comparaciones entre sexos en las variables medidas por el cuestionario. Los resultados obtenidos confirman lo encontrado en estudios anteriores (Merisuo-Storm y Soininen, 2012; Villalón et al., 2014) observándose diferencias significativas, a favor de las niñas, en la puntuación global del cuestionario, así como en la motivación hacia la lectura y los intereses lectores. Estas diferencias en los intereses lectores parecen estar 
especialmente relacionadas con la lectura recreativa. No se observan diferencias significativas entre los grupos en lo que respecta a la actitud hacia el estudio.

Además, al igual que en estudios realizados en otros contextos -Merisuo-Storm (2006) con alumnado finlandés; Eriksson (2002) con estudiantes suecos; y Gambrell (1996) en los Estados Unidos- se observan diferencias significativas en la lectura social, que indican que las niñas muestran mayor disposición hacia la lectura en voz alta y compartida, mientras que los varones tienden a mostrarse reacios ante este tipo de lectura. Muchos varones declaran que odian leer en voz alta y se sienten incómodos y ansiosos cuando se les requiere leer en público, incluso aunque lean bien. Una posible hipótesis para explicar estos resultados podría ser que los varones se ven más presionados por la opinión de sus compañeros y les preocupa más la aprobación por parte de estos. Aerila y Merisuo-Storm (2017), en un interesante artículo dirigido a cómo lograr que los niños disfruten de la lectura, sugieren varias soluciones efectivas para este problema. La primera, que los estudiantes más mayores lean en voz alta a los más pequeños. De esta forma tendrán la oportunidad de leer libros menos difíciles de los que normalmente leen en sus clases y, dado que los estudiantes más jóvenes no criticarán su forma de leer, pueden fortalecer su auto-confianza y mejorar la percepción que tienen de sí mismos como lectores. Una segunda sugerencia es que los niños pequeños le lean en voz alta a un animal de compañía. Por lo general los niños encuentran que su perro puede ser un oyente más fácil que sus compañeros o que sus profesores. Este escucha pacientemente, sin juzgar, incluso aunque se equivoque o lea despacio. Otra forma de conseguir que los niños lean más y disfruten de la lectura es montando una representación de teatro para la cual los alumnos tengan que leer y ensayar repetidamente un texto. Con estas lecturas repetidas durante los ensayos, su lectura se vuelve más eficiente. Asimismo, leer en parejas puede ser una buena estrategia: de esta forma pueden compartir los aspectos cómicos de la lectura así como sus opiniones sobre la misma. En concreto, Aerila y Merisuo-Storm (2017) señalan que el leer en parejas mixtas, junto a una niña, puede ayudar a los varones a desarrollar una mayor sensibilidad hacia la lectura y a empezar a comprender mejor la personalidad y los sentimientos de los personajes. Finalmente, recomiendan también las visitas de los abuelos a la escuela para leer a los niños, dado que la experiencia muestra que los niños disfrutan mucho de estas lecturas y además ofrece la oportunidad de que los niños se expongan a un modelo lector masculino.

Un dato que llama la atención es que, curiosamente, a pesar de que la mayor parte de los estudios muestran que, en general, las niñas tienen mayor competencia lectora que los niños, podemos observar en nuestro estudio que los varones tienen una mejor percepción de la competencia lectora que las niñas, indicando una mejor imagen de sí mismos como lectores y una mayor confianza en su competencia que las niñas. No parece existir una explicación clara de a qué pueden atribuirse estas diferencias: no 
obstante, se trata de una distorsión de la realidad que debe ser estudiada si se quieren poner en marcha posibles mecanismos correctores.

En definitiva, se presenta un instrumento que puede ser de utilidad al profesorado y otros profesionales de la educación para identificar las actitudes e intereses de niños y niñas hacia la lectura, tanto en el contexto académico como en el recreativo. Este cuestionario, de fácil aplicación, puede ser administrado por el docente a toda la clase con la finalidad de tomar decisiones a la hora de planificar la instrucción o bien para evaluar las actitudes e intereses de sujetos específicos, así como para evaluar los cambios producidos en un grupo como resultado de la aplicación de determinados programas de instrucción. La identificación de actitudes negativas hacia la lectura en estudiantes concretos puede sugerir la necesidad de profundizar sobre la naturaleza y el origen de dichas actitudes a través de otros métodos como la observación, la entrevista estructurada o los inventarios de intereses.

De los resultados pueden extraerse interesantes implicaciones educativas para los maestros y las maestras. La comprensión de las actitudes y preferencias de los estudiantes puede proporcionar a los educadores información valiosa de cara a diseñar programas y materiales que tengan en cuenta los intereses y las necesidades de los participantes. Para conseguir motivar a todo el alumnado, los profesores deben indagar en cuáles son los temas y géneros de literatura que pueden interesar a los estudiantes más reacios hacia la lectura (Soininen, Merisuo-Storm, y Korhonen, 2013). A su vez, deben proponer una amplia variedad de textos y de actividades relacionadas con la lectura de tal forma que los intereses, tanto de niños como de niñas, puedan ser cubiertos.

Además, deben trabajar conjuntamente con las familias para fomentar actitudes positivas hacia la lectura, teniendo en cuenta, como señalan Andrés, Urquijo, Navarro y García-Sedeño (2010), que un contexto familiar alfabetizador potencia la adquisición de ciertas habilidades prelectoras, las cuales, a su vez, predisponen al niño a un desempeño lector favorable y exitoso; en este sentido, Alves, Assis, Martins y Da Silva (2017) destacan el importante impacto de la familia en el rendimiento académico y en el desempeño cognitivo en estas edades; asimismo, la motivación por los libros y la actitud lectora del alumnado que comienza la etapa de Primaria puede aumentar de manera significativa si se llevan a cabo programas de implicación familiar en la lectura (Mora, Galán y López, 2016).

Los profesionales de la educación contribuirán a fomentar en los estudiantes una actitud más positiva y, en definitiva, a que se conviertan en lectores motivados y autónomos, si les permiten elegir aquellos libros que más les interesan. También se debería permitir a los niños dar su opinión sobre los libros seleccionados por el profesor. Además, parece aconsejable exponer a éstos una gran variedad de géneros y textos para que puedan desarrollar una amplia variedad de destrezas y estrategias que sean aplicables a la lectura en la vida real (Mohr, 2006). Cuando se les permite leer materiales 
que tienen una relevancia para su vida cotidiana, los niños se convierten en lectores motivados.

Aerila y Merisuo-Storm (2017) señalan que los niños necesitan disponer de rincones o lugares de lectura donde puedan leer sin distracciones, lugares agradables donde puedan leer, solos o con un compañero, y donde dispongan de la suficiente privacidad y tranquilidad a la hora de leer.

Finalmente, en futuros estudios convendría confirmar estos resultados contando con una muestra más representativa: a través de un muestreo aleatorio, con la participación de colegios públicos y de centros educativos pertenecientes a otras comunidades autónomas, además de la de Madrid. Asimismo, sería interesante investigar el rol que desempeña el profesorado en la elección de los libros por parte del alumnado, así como desarrollar más trabajos sobre los estereotipos de género, ya que tanto este estudio como otras investigaciones revisadas (Chapman, Filipenko, McTavish y Saphiro, 2007), muestran que existen diferencias en el tipo de libros que niños y niñas consideran como más apropiados.

\section{REFERENCIAS}

Aerila, J.A., y Merisuo-Storm (2017). Emergent readers and the joy of reading: A Finnish perspective. Creative Education, 8, 2485-2500. doi 10.4236/ce.2017.815171.

Aguilar, M., Aragón, E., Navarro, J.I., Delgado, C. y Marchena, E. (2017). Análisis del efecto san Mateo en un estudio longitudinal sobre el desarrollo lector durante la educación primaria $\left(1^{\circ}\right.$ a $\left.5^{\circ}\right)$. European Journal of Education and Psychology, 10(1), 23-32.

Ainley, M., Hillman, K., y Hidi, S. (2002). Gender and interest processes in response to literary texts: Situational and individual interest. Learning and Instruction, 12(4), 411-428.

Alves, A.F., Assis, C.M., Martins, A. y Da Silva, L. (2017). Desempeño cognitivo y rendimiento académico: ¿cómo confluyen familia y escuela? European Journal of Education and Psychology, 10(2), 49-56.

Andrés, M., Urquijo, S., Navarro, J., y García-Sedeño, M. (2010). Contexto alfabetizador familiar: relaciones con la adquisición de habilidades prelectoras y desempeño lector. European Journal of Education and Psychology, 3(1), 129-140.

Artola, T., Sastre, S., y Barraca, J. (2017). Diferencias de género en actitudes e intereses lectores: una investigación con alumnos españoles de primaria. Bordón. Revista de Pedagogía, 68(2).

Asún, R.A., Rdz-Navarro, K., y Alvarado, J.M. (2016). Developing multidimensional Likert scales using item factor analysis: The case of four-point items. Sociological Methods \& Research, 45(1), 109-133.

Boltz, R. (2007). What we want: Boys and girls talk about reading. School Libarary Media Research, 10. 
Cartwright, K.B., Marshall, T.R., y Wray, E.A. (2015). Longitudinal Study of the Role of Reading Motivation in Primary Students' Reading Comprehension: Implications for a Less Simple View of Reading. Reading Psychology, 37(1), 55-91. doi: 10.1080/02702711.2014.

Chapman, M., Filipenko, M., McTavish, M. y Saphiro, J. (2007). First graders'́preferences for narrative and/or information books and perceptions of other boys'and girls'book preferences. Canadian Journal of Education 30(2), 531-553. doi: 10.2307/20466549.

Chick, K., y Heilman-Houser, R.A. (2000). Children's literature choices: Gender stereotypes prevail. Pennsylvania Reads: Journal of the Keystone State Reading Association, 1(2), 3-13.

Cooter, R.B. y Alexander, J. (1984). Interest and Attitude: Affective Connections for Gifted and Talented Readers. Reading World, v24 n1 p97-102 Oct 1984 Association, 1(2), 3-13.

Elosua, M.R. y Gómez-Veiga, I. (2014). Habilidades lectoras y rendimiento académico en $3^{\circ}$ y $6^{\circ}$ de Primaria: aspectos evolutivos y educativos. Estudios de Psicología, enero. doi: 10.1174/0210993912800676411.

Eriksson, K. (2002). Booktalk Dilemmas: teachers' organisation of pupils' reading. Scandinavian Journal of Educational Research, 46(4), 391-408.

Gambrell, L.B. (1996). Creating classroom cultures that foster reading motivation. The Reading Teacher, 50(1), 14.

Gómez-Benito, J., Hidalgo, M.D., y Guilera, G. (2010). El sesgo de los instrumentos de medición. Tests justos. Papeles del Psicólogo, 31(1), 75-84.

Graham, S., Berninger, V., y Abbott, R. (2012). Are attitudes toward writing and reading separable constructs? A study with primary grade children. Reading \& Writing Quarterly, 28(1), 51-69.

Green, S.B., y Yang, Y. (2015). Evaluation of dimensionality in the assessment of internal consistency reliability: Coefficient alpha and omega coefficients. Educational Measurement: Issues and Practice, 34(4), 14-20.

Hu, L.T., y Bentler, P.M. (1999). Cutoff criteria for fit indexes in covariance structure analysis: Conventional criteria versus new alternatives. Structural equation modeling: $a$ multidisciplinary journal, 6(1), 51-55.

Marinak, B.A., y Gambrell, L.B. (2010). Reading motivation: Exploring the elementary gender gap. Literacy Research and Instruction, 49(2), 129-141.

McKenna, M.C., y Kear, D.J. (1990). Measuring attitude toward reading: A new tool for teachers. The reading teacher, 43(9), 626-639.

Merisuo-Storm, T. (2006). Girls and boys like to read and write different texts. Scandinavian Journal of Educational Research, 50(2), 111-125.

Merisuo-Storm, T., y Soininen, M. (2012). Young boy`s opinions about reading, literacy lessons and their reading competence. In ICERI2012 Proceedings (pp. 4109-4118). IATED.

Merisuo-Storm, T., y Soininen, M. (2014). Interesting reading materials and excercises encourage also reluctant boys to read. Social and Behavioral Sciences, 116, 2583-2588. doi:10.1016/j.sbspro.2014.01.615.

Mohr, K. (2006). Children's choices for recreational reading: A three-part investigation of selection, preferences, rationales, and processes. Journal of Literacy Research, 38(1), 181-204. 
Mora, J., Galán, A., y López, M. (2016). Eficacia de un programa de implicación familiar en la lectura de alumnado de $1^{\circ}$ de Educación Primaria. Revista de Psicodidáctica, 21(2), 375-391.

Mullis, I.V., Martin, M.O., Foy, P., y Drucker, K.T. (2012). PIRLS 2011 International Results in Reading. International Association for the Evaluation of Educational Achievement. Herengracht 487, Amsterdam, 1017 BT. The Netherlands.

Oakhill, J.V., y Petrides, A. (2007). Sex differences in the effects of interest on boys' and girls' reading comprehension. British Journal of Psychology, 98(2), 223-235.

Pestcher, Y. (2010). A meta-analysis of the relationship between students' attitudes towards reading and achievement in reading. Journal of Research in Reading, 33(4), 335-355.

Satorra, A. (2000). Scaled and adjusted restricted tests in multi-sample analysis of moment structures. In Heijmans, R.D.H., Pollock, D.S.G. y Satorra, A. (eds.), Innovations in multivariate statistical analysis. A Festschrift for Heinz Neudecker (pp.233-247). London: Kluwer Academic Publishers.

Sadowski, M. (2010). Putting the "boy crisis" in context. Education Digest: Essentil Readings Condensed for Quick review, 76(3), 10-13.

Soininen, M., Merisuo-Storm, T., y Korhonen, R. (2013). What kind of competence teachers need in the year 2020? Profesorado. Revista de Currículum y Formación de Profesorado, 17(3), 111-122.

Stevens, S. (2011). Entering bookworld: How to help both Boys and Girls in Class Read Alouds. Teacher as Leader, EDU 600, 1-9.

Stutz, F., Schaffner, E., y Schiefele, U. (2016). Relations among reading motivation, reading amount, and reading comprehension in the early elementary grades. Learning and Individual Differences (45), 101-113. doi.org/10.1016/j.lindif.2015.11.022.

Schwartz, W. (2002). Helping underachieving boys read well and often. New York: ERIC Digest.

Sullivan, M. (2004). Why Johnny wont read. School Library Journal, 50(8), 36-39.

Trizano-Hermosilla, I., y Alvarado, J.M. (2016). Best alternatives to Cronbach's alpha reliability in realistic conditions: congeneric and asymmetrical measurements. Frontiers in psychology, 7, 769 .

Villalón, R., Melero, A., e Izquierdo-Magaldi, B. (2014). Spanish Primary School pupil`s Literacy attitudes: are they related to their reading comprehension and their feeling of competence? In ICERI2014 Proceedings (pp. 36-41). IATED.

Villalón, R., Merisuo-Storm, T., e Izquierdo-Magaldi, B. (2015). ¿Importa la actitud hacia la lectura a la hora de comprender un texto? $3^{\text {rd }}$ International Congress of Educational Sciences and Development. San Sebastián, Spain. doi: 10.13140/RG 2.1.1936.7524.

Villalón, R., Melero, A., e Izquierdo-Magaldi, B. (2016). Reading attitudes and text comprehension of Finnish and Spanish Pupils: a comparative study. EARLI SIG-2 conference. Geneva, Switzerland.

Watson, A.K., Kehler, M. y Martino, W. (2010). The problem of boy's literacy underachievement: raising some questions. Journal of Adolescent \& Adult Literacy, 53(5), 356-361. 


\section{ANEXO I}

\section{Adaptación al castellano del Cuestionario de Merisuo-Storm y Soininen}

Presentación y formato de respuesta:
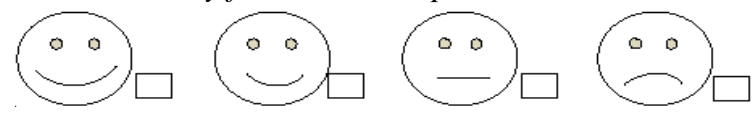

Relación de preguntas incluidas en el cuestionario:

1.- ¿Te gusta leer libros?

2.- ¿Te gusta leer libros que sean de cuentos?

3.- ¿Te gusta leer libros de aventuras?

4.- $¿$ Te gustan los libros que tienen muchos dibujos?

5.- ¿Te gusta que los personajes de los cuentos sean buenos y felices?

6.- $¿$ Te gusta leer en casa en tus ratos libres?

7.- ¿Te gusta ir a la biblioteca?

8.- ¿Te gustó aprender a leer?

9.- ¿Te gustó aprender a escribir?

10.- ¿Te gusta hacer ejercicios y actividades sobre algo que has leído?

11.- ¿Te gusta hacer deberes o tareas escolares en casa?

12.- ¿Te gusta hablar de los libros que lees con tus amigos?

13.- ¿Te gusta hacer los trabajos y actividades de clase con un/a compañero/a?

14.- ¿Te gusta leer en voz alta en clase?

15.- ¿Te gusta contarles el libro que estás leyendo a otros compañeros?

16.- $i$ Te gusta leer con un/a compañero/a?

17.- ¿Te resulta fácil leer?

18.- ¿Te resultó fácil aprender a leer?

19.- ¿Te resulta fácil entender los libros que lees en el colegio?

20.- ¿Entiendes bien las palabras que lees?

21.- ¿Te resulta fácil acordarte de lo que lees en el colegio? 